\title{
The establishment of integrated Chinese and western "precision medicine"- modern medicine towards complexity science age
}

\begin{abstract}
A medical system that has become the mainstream medicine does not just depend on the medicine itself, but on the mainstream culture and science concepts at that time in history. In times of natural science develop from simple science to complexity science, medical field is inevitably get involved. "Precision Medicine" plan is an initial attempt of medicine towards complexity. Dealing with a complex life system of human body, modern Western medicine is trying to establish a body states description system along the "analysis-reconstruction" way from micro-level perspective. Traditional Chinese Medicine establishes a body states description system from the holistic level by means of metaphor and analogy. Thus, in times of complexity science, modern medicine will integrate Chinese and Western medicine that towards "precision". Through different levels of + from various directions, it will deepening our understanding of human life, and enhances our ability of medical disease control.
\end{abstract}

Keywords: complexity science, precision medicine, traditional chinese medicine, modern medicine, personalized medicine
Volume 4 Issue I - 2016

\author{
Bing Yuan \\ Hong Kong Eastern Medicine Research Centre, China
}

Correspondence: Bing Yuan, Hong Kong Eastern Medicine Research Centre, Flat A-B, I2/F, Bonds Mansion, 554-556 Nathan Road, Kowloon, Hong Kong, China, Tel +852 2300 1898, Email greenisland@vip.163.com

Received: May 15, 2016| Published: June 23, 2016

\section{Introduction}

Since the $21^{\text {st }}$ century, with the development of complexity science and the advancement in computer information technology, there are unprecedented vision and technological means for mankind: the most advanced product of biological evolution on this planet. The success of the Human Genome Project opens up a new way for a more comprehensive understanding of modern medicine and the treatment of human diseases. In this circumstance, Precision Medicine is proposed by the American scientific community first. Advocated by the heads of states of the world's two most powerful economies - the USA and China - as well as the medical professionals worldwide, Precision Medicine quickly becomes the hot topic of common concern everywhere.

Modern medicine moves towards personalized medicine: Precision Medicine. Traditional Chinese Medicine (TCM) has more than two thousand years of history. Does it need precision? If yes, how it will become precision? What is the relationship between TCM and modern medicine as far as Precision Medicine is concerned? What role does TCM play in modern medicine of the $21^{\text {st }}$ century? Today, it becomes the focal problems for the medical profession.

\section{The impact of civilization and science on} mainstream medicine

Throughout the clinical practices of the Chinese and Western medicines, most diseases diagnosed by Western medicine, the treatment is basically limited to control targets, to remove lesion, and to kill pathogens. In most cases, the side effects are unavoidable. Although there is no clear disease diagnosis, TCM diagnosis and treatment are according to syndromes, it is a holistic treatment which may achieve good results with lesser side effects as compared to the treatment by Western medicine.

Today, TCM and acupuncture therapy have come out of China and is saw around the world. No matter one belongs to the medical professional or from general public, in China or in the Western countries, it has become a general consensus concern about the problems faced by modern medicine as well as TCM. Though Western medicine is established based on the analytical methodology of static decomposition and one-factor analysis, it is still be regarded as modern medicine and firmly occupies the position of mainstream medicine. Although more and more people have come to realize the setbacks of Western medicine and are turning to TCM for cure and treatment, TCM is considered scientifically heterogeneous -an oldfashioned medicine. TCM is being marginalized. It is still considered illegitimate practice in many parts of the world.

For this reason, many people feel perplexed, helpless, and angry. In fact, looking back at the history of medicine, we know it very well that a medical system that has become the mainstream medicine does not just depend on the medicine itself, but on the mainstream culture and science concepts at that time in history.

No matter it was in the East or West, holism was the dominant philosophy of ancient times. Holism of traditional medicine had been among the mainstream medicine. In Europe, Aristotle ( $3^{\text {rd }}$ century $\mathrm{BC})$ and Galen $\left(2^{\text {nd }}\right.$ century $\left.\mathrm{AD}\right)$ had made great achievements in anatomy and physiological anatomy. They began the application of experimental analysis. ${ }^{1}$ But they still could not shake the dominance of mainstream holistic medicine.

Modern medicine was originated in Europe. After the second half of the $15^{\text {th }}$ century along with the beginning of the Renaissance in Europe, the establishment of the $17^{\text {th }}$ century Newtonian physics system, and the first industrial revolution began in the mid- $18^{\text {th }}$ century, there was a complete change in the perception of the understanding of the world. With the rise of the mechanistic view of nature, the holistic form of exercise was replaced by low-level form of exercise, enabling reductionist scientific method to become the mainstream method. Anatomical decomposition and one-factor analysis medical methods were deemed to meet the contemporary concept of science. It gradually developed to become a scientific analysis methodology of 
the medical system. It occupied the position of mainstream medicine. The holistic approach of traditional medicine was considered to be unscientific, was marginalized, and gradually faded out from the stage of history.

In China, Western medicine came into the scene one hundred years ago. Since the Opium War in 1840, following the invasion of foreign powers, Western medicine entered China as a by-product of Western civilization and science. Defeated in the Sino-Japanese war in 1895 , Chinese people was introduced the concept of Western science and technology as well as Western civilization. As Western scientific ideas were becoming the mainstream scientific ideas, Western medicine replaced TCM though TCM had two thousand years of history in China. Western medicine became the mainstream medicine in China and the status quo continued until today. TCM was developed from five thousand years of Chinese civilization and it had become one the world's most powerful traditional medicine systems. It had played a significant role in the process of multiplication and prosperity throughout all the various Chinese dynasties. It had created numerous medical miracles that still could amaze the medical science of today. However, it still could not escape the fate of being suppressed, marginalized and some of the know-how were even abolished.

After the beginning of the 20th century, with the development of natural science, the scientific method based on analytical methodology seems to come to its end. Science begins to directly face the complexity of the world. The scientific research theory returns to the holistic approach. The concept of a theoretical physicist and Oriental philosophy of ancient sages are becoming more consistent on understanding the origin of the world and the origin of the universe. Clearly, the concept of science to the concept of civilization is undergoing a subtle but significant change. It indicates that our history is in the eve of a big change like the Renaissance of Europe before. This change originated in the birthplace of the Renaissance hundreds of years ago, is also from the West- the leading trend of today's technology and civilization: U.S.A. and Europe.

This change first appeared in the scientific forefront of theoretical physics. From the early 20th century, Albert Einstein's general theory of relativity, Planck's quantum mechanics, to Stephen Hawking's quantum theory of gravity, the understanding of the origin of the world tends to unify with the traditional Oriental philosophy. No matter they be today's contemporary masters or the oriental ancient sages, they understand that the universe and the composition of its various parts are closely related to the whole. The universe is self-consistent, the whole and its part contains each other. The world is the unity of matter and energy. Consciousness and substances can be transformed into energy. The substance does not objectively exist, its status changes with observers. Past, present and future, are just the illusions that exist in people's minds. ${ }^{2}$

20th century science, on the one hand, in the traditional areas of science - as represented by theoretical physics-is from micro to cosmoscopic and deepening human's understanding of the origin of the world. On the other hand, along with systems theory, cybernetics, information theory, theory of dissipative structures, synergetic, and mutation theory are expanding the territory and horizon of science. ${ }^{3}$ After the 1970s, with the surge of complexity science, scientists would no longer divide one live complex object study into pieces of dead part separate studies. They faced complexity directly and treated it as a live organic whole. They used metaphor and analogy to build model, through the behaviour observation and simulation as a whole, to grasp and understand its inherent laws.
Scientific concept changes according to the advancement of science theory. It spreads to applications which will inevitably affect the idea of modern civilization. Scientists begin to see the world as an organic whole. They begin to realize that science has limitations and drawbacks. They understand the purpose of nature. The idea of transformation of nature is gradually giving way to better harmony with nature, and return to nature. At the same time, Industry 4.0, the fourth industrial revolution starts. It is a reversal of the previous three industrial revolutions - on scale and batch production development mode. Overcoming most hurdles in manufacturing, it proposes a new idea and concept of personalized manufacturing for individual and particular order. With IoT and artificial intelligence technology, the traditional business model will soon be replaced. The recent popular internet shopping network, in the near future, will become the mainstream and trend of business model.

In this background, modern medicine as an applied science under modern scientific system has to comply with modern civilization and modern science theme. It has to adjust the context of medical concepts and development. In the 1980 s, there were calls for changes in the medical model- from the past medical model mechanistic, now the biomedical model, to the future bio-psycho-social medical model. Recent years, with completion of Human Genome Project plus the development of artificial intelligence as well as the arrival of big data analysis, the world's leading science and technology of the American scientific community first proposed a personalized medicine - the core of Precision Medicine. It is a meaningful initial step for analytical methodological medicine to holistic medicine.

\section{Modern medicine towards precision medicine}

In 2011, the National Academy of Sciences, the American Academy of Engineering, National Institutes of Health, and the National Science Board jointly proposed the Toward Precision Medicine initiative. Dr. Maynard V. Olson, the famous Genome scientist, involved in the drafting of the US National Intelligence Report published a report titled "Toward Precision Medicine". This report presented a genetic study, and tight integration with clinical medicine, aimed at achieving the precise treatment of human disease and raised the alarm of its adverse effects.

Precision Medicine refers to the development of a customized medical model that personalizes treatment programs based on the unique characteristics of each patient. Obviously, Precision is not limited to our conventional understanding of the meanings of being accurate and precise. It is the overall status of the human body being precisely understood. ${ }^{4}$ American Precision Medicine program aims at tumour therapy as the short-term goal. Its long-term goal is to expand its application to all other areas of health care. NIH Director, Francis Collins, when interpreting President Obama's Precision Medicine plan, said that he envisioned a grand scale to collect one million or more volunteers involved in the large databases study. He said that participants would be required to render biological data as specimens including: the number of cells, proteins, metabolites, RNA and DNA, full genome sequencing, behavioural data, and electronic health records.

Obviously, the US scientists use big data analysis -with extensive statistical correlation between the various measurable indicators of human body in a variety of diseases - in order to reflect the overall status of the human body. It is the foundation of a more in-depth, accurate and comprehensive description of essential characteristics of the human body. 
Precision requirements of personalized medicine, for specific body part or for diseases caused by specific elements, is the accurate positioning of the disease and the accurate treatment with precisely researched target drugs. However, for complex diseases involving multiple tissues and organs abnormality, it is difficult to attribute the disease to specific abnormal organs and tissues. Case like this requires a system that can fully describe or reflect the state of the overall status of the body. And with such a precise description of the overall status, it can precisely comprehend the status that is defined by a plurality of state variables (or test indicators).

Using the status description system to identify the body condition, especially for precise diagnosis, is related to the precision of system status variables detection, and to associate them with related human disease precisely. It is also related to the integrity of the status variables and their degree of abstraction as well as their total number. We want to improve the detection accuracy of the detection equipment or instrument to find the status variables that are associated with human diseases more accurately. Meanwhile, we must ensure the status variables introduced cover the basic physiological and pathological human activities, i.e., to ensure the integrity of the system status variables. In addition, the human body from macro to the micro level division -the system, organs, tissues, cells, and molecules - the status variables so introduced belong to different levels. To fully describe the magnitude of the body status, the required amount of status variables, are closely related to the accuracy of the description of the human body.

Generally speaking, the lower the level of status variables require the greater total number of variables to fully express the body's physiological and pathological activities. On the contrary, the higher level of status variables gives a better level of abstraction and requires smaller number of variables. From the perspective of precise drug research point of view, drugs that are produced according to controlled macroscopic status variables research may function relatively broader coverage. It will cover the micro level and has the sum of direct or indirect effects of multiple variables. From the control point of view, the more macroscopic status variables are the better the abstract, the simpler to describe the state of the system, and easier to understand and to control. Thus, the precision requirements to identify the body status will be lesser. The accurate requirements of understanding the role of drugs will be reduced based on the macroscopic status variables research. If we were to observe a soldier's beard on the ground from an orbiting satellite, we need a high-powered telescope. But if we were to observe a mountain or a river, we do not need to be so accurate. Similarly, if the missile aims at a vehicle, using the energy of ordinary shells, of course, it needs precise positioning and precise control trajectory. But if we use a bomb to bombard a city, then the accuracy of positioning and control will be greatly reduced.

Both from the Precision Medicine initiative of the United States and the Precision Medicine project brewing in Mainland China, the biological specimens so collected such as: the number of cells, proteins, metabolites, RNA and DNA, and full genome sequencing are quite microscopic. That is, at this level, the establishment of the state of the overall system description means higher accuracy and consist a huge amount of data. Therefore, whether it is to achieve precision diagnosis test, precision drug research, or precision control of human body, we have a long way to go. We are on the right path to change from microscopic analysis to holistic integration development.

Western medicine, from its formation to today's scale of development, experienced a long history of about 300years. Every discovery or development resulted a great leap forward in the diagnosis and treatment of human diseases. Today, it is still a long and winding road to develop from microscopic analysis to holistic integration. However, today's technological means, the speed of knowledge accumulation, and data handling capability are far more advance than before. And every discovery or development by medical scientists improves our ability to deal with some of the medical causes of human disease. It is expected, with the passage of time, the medical methodology that dominated for the past three hundred years will soon be gradually abandoned. A new and quickly established body status description system will bring modern science to a new horizon.

\section{Traditional chinese medicine also need and be able towards precision}

Precision Medicine embodies the concept of personalized medicine. It is the same as the TCM's theories of treatment according to person, locality and time, and syndrome differentiation and treatment. This is aligned with the trend of modern civilization and modern science revisiting ancient civilizations and ancient science of the orient. In the wake of the trend of personalized medicine, modern medicine returns to the concept of oriental medicine. What will happen to the oriental medicine? What will happen to TCM?

From the perspective of modern medicine, the complexity science will gradually become the mainstream of scientific concepts. With the development of Precision Medicine, the body status description system of personalized medicine will gradually establish and becoming mature. Thus, modern medicine will not only be evidence based medicine, but also has a body status medical model similar to TCM. Based on this body status medical model, in the course of two thousand years, TCM could fight against diseases, and built up experience of medical syndromes identification as well as diagnosis and treatment according to syndromes. It will be included in modern medicine with a different formulation of body status medical system. Like Newtonian mechanics that revealed the laws of nature, it still exists with a change in form and representation in relativistic mechanics, of course, it may require at least a few years or even one or two hundred years of long and difficult exploration. Thus, TCM, a medical system that has a long history of two thousand years, may not TCM not continue to exist with any practical significance at all. It may only exist only in historical text of medicine or as a teaching reference with similar fate like that of Newtonian physics.

If we take TCM, not as a legacy, but as a developing science, the situation would be quite different.

TCM's diagnosis and treatment according to syndromes system, as a system to describe body states and control, perfectly embodies personalized medicine - the core of Precision Medicine. TCM has the core elements of Precision Medicine. In this case, on the road towards Precision Medicine or personalized medicine, TCM is unique and it took the initiative. For TCM to achieve the goal of Precision Medicine, it is just a technical issue of precise syndrome differentiation, precise drug and precise treatment. ${ }^{6}$

To achieve precision of TCM's diagnosis and treatment according to syndromes system, the first issue is to make TCM's human body status description system, i.e., the syndrome differentiation system be more precise. It mainly includes three aspects. Firstly, scientific and standardization of TCM theories, that is, to develop body status description system based on logical rules of strict science. ${ }^{7}$ Secondly, minimize the impact of subjective factors from practitioners and patients in the information collection process. Introduce research indicators that have good correlation with human body status. Thirdly, 
more precise and measurable indicators by using artificial intelligence and pattern recognition techniques as well as statistical data based on the big data analysis. It is basically the same starting point as syndromes normalization at the beginning of the 1980s.

In the early eighties, alongside with the tides of China's four modernizations, Chinese medical practitioners proposed "TCM Modernization". The TCM practitioners realized that TCM's fundamental principles and syndrome differentiation and treatment forms the basis of TCM modernization and shall also form the research reference for the modernization of Chinese medicine. Therefore, standardization of TCM's diagnosis and treatment according to syndromes has been put on the table. It began to focus on the standardization of syndromes and clinical symptoms. Related books were published.

Today, from the perspective of the history of science, when we look back on its course and to analyze the underlying cause making it unsuccessful, we note the following.

a. Standardization was only limited to the syndrome level. No issue on the top TCM theoretical structure of syndrome determinant set. There was no scientific and standardization on TCM's theory. Thus, there was no uniform principle to follow in syndromes definition and setup. Different syndromes standard was developed by different experts. There is no unified planning and construction principles of the entire syndrome differentiate system. Finally, it was just a collection of expertise convergence. Unavoidable logical confusion existed but no rigorous scientific system was created.

b. Chinese medicine sector at that time was not as strict as science researches. There was no scientific research methods and rules. There was no mechanism to introduce the theoretical basis of clinical observation verification, revision and improvement, as the scientific theory development did.

c. Many qualitative issues can be better solved by ways of quantitative algorithms. Merely stay at the qualitative level without any quantitative algorithms and not utilizing the progress of fuzzy recognition technology may hinder progress.

If this attempt ever fails, it will seriously set back the modernization of TCM. TCM and its detection methodology cannot proceed nor progress because there are no terms of reference. And, it is inevitable modern medicine will be the only terms of reference for human body and disease descriptions.

Today, the advancement of scientific progress, its technology, and concept are quite different from 30years ago. The development of complexity science confirms the comparative images approach used by ancient Chinese medical practitioners was identical to today's complexity science research method. Since complexity science is a science, it follows that the basic scientific principles of analytical methodology prevails. It also follows that the general mechanism of scientific theory establishment, to develop, to improve, and to abandon will also exist. Today, the rapid development of information technology, artificial intelligence, and pattern recognition technology are well developed and widely used. The successful arrival of the era of big data analysis and the human genome project, based on the TCM theory system, the establishment of precise description of human body status has sufficient technical basis. This brings us to stand on an unprecedented modern technology podium.

All the Chinese medicine practitioners are at the cross road to make a choice: to treat TCM as a legacy or a science. If, as a legacy, what we need is inherited intact. If, as a science, it is necessary to introduce a general mechanism of scientific development - the construction of the theoretical basis of observation and experiment, using controlled experiments to test its theory, and to revise and improve the theory according to its results. The development of the theory is improved by practical tests.

With the precise human body status description system as a frame of reference, we can observe through experiments and statistical analysis, and apply the precise drug, and thus actualize the precision treatments for human disease.

We've talked about the hierarchy, the status variable's level of abstraction, its requirements and impact on the identification and control precision. For identification, the higher level of abstraction, require the less total number of variables. The human body status description system will be simple; can be easily understood and controlled. And the degree of precision so required will be relatively low by using it to identify the body status. Precision drug based on controlled macroscopic status variables research will be relative macro. It has broader coverage, including the micro level - the sum of direct and indirect effects from multiple variables. For control, it makes precision requirements to understand the drug's function being reduced.

The micro level variable precision does not mean the precise description of human body status. By the same token, the higher the variable level, the higher precision of showing the overall human body status. Obviously, the precision control of human body is closely related to the level of variables we have to control. The higher the level, the lower required precision, and the smaller side effects of drugs created. The reverse is true. This is why when we study TCM and Western medicine, we find that TCM herbs are very vague, but are good enough for TCM clinical usage. Western drugs functions are in great detail, from a higher level perspective, there is more uncertainty and side effects.

In TCM theory, the human physiological and pathological activities defined by functional division of holistic level. In recent years, medical research of body constitutional medicine shows that there are 10 kinds of body constitutions - derived from TCM theory - can represent the different but relatively stable human body status. By way of syndrome differentiation and treatment, TCM defines 100 kinds of fundamental syndromes. It covers all basic pathological processes of human body. Obviously, TCM body status description systems are the top level of abstraction. It is the highest level. The required level of accuracy of diagnosis and drug research is lower, and resulted macro drugs with long-term performance. It means that from a technical perspective, TCM is closer to the goal of Precision Medicine. In other words, the works to be done are much less and easier to achieve this goal. In short, TCM stays ahead of other medical science in moving towards Precision Medicine.

\section{The structure of precision medicine and modern medicine based on the integration of chinese $\&$ western medicine}

In the extensive continuous study after the completion of the Human Genome Project, scientists firmly grasp the core objective Complex Systems Biology (CSB). On the one hand, thousands genes or proteins are found via high-throughput and large-scale Omics Analysis. On the other hand, reveal the relationship and function of these components by applying existing experimental scientific method. Scientists' goal is to establish mathematical models of 
biological systems by getting massive experimental data. It can describe the structure of the system and its response to external action through analysis and calculation. Ultimately achieve the micro level and the overall macro-life integration.

In this sense, medical profession had shaken off the shackles of analytical methodology that ruling the modern medicine for 300 years. Modern medicine began to establish a describe system of the human body holistic status, to achieve personalized medicine attempt. Today, books or textbooks that talk about TCM state the core of TCM is the concept of holism and diagnosis and treatment according to syndromes. CSB and personalized medicine is exactly the modern version of the TCM's core features. That is, along with the progress of modern medicine towards holism and personalized medicine, the concept of holism and diagnosis and treatment according to syndromes will no longer be the distinguishing features of the Western or TCM medical systems.

Obviously, complexity science and Precision Medicine is the main science trend. The idea of modern medicine is returning to the idea of TCM. We've also talked about TCM needs and able to be precision. If TCM become scientific -introduce mechanisms regulate the development of science and scientific theory - TCM will be a precision science subject appear on modern medicine of complexity science basis. In future modern medicine framework, there will be Precision Medicine based on existing modern medicine, and based on TCM. What is their difference then?

The most fundamental difference between the two bases mentioned above is the different in level of human body status description systems. Based on scientific holism, TCM describe human body status of physiological and pathological activities at the highest level. Based on scientific reductionism, modern medicine decomposes human body into the molecular level. Genetic medicine, proteomics, and modern medicine are trying to establish the overall human body status description system in the smallest unit of life characterized by molecular level. We know that the total number of cells of the human body is about 60 trillion. At molecular level, the total number of components will increase in certain magnitude.

Of course, it is impossible for modern medicine to describe human body status by molecule or cell parameters. Even with massive simplification, it is still astronomical in human body status description parameters when comparing to TCM's systems: five viscera and six bowels, dialectical fluid blood, 2-digits numbers of body constitution, and 3-digits numbers of syndromes. Research on the human genome found that there are hundreds of millions of gene variants. In preliminary correlation analysis of genes associated with human diseases, scientists have clearly recognized that the majority of human disease is not decisively related to genes. It will involve protein, the product of metabolism and so on. Obviously, to establish a complete description of the human condition system at this level, regarding to the total number of parameters introduced, it takes at least several orders of magnitude to measure the different from holistic level.

In physics, from macro to micro material hierarchy chain - from galaxies of the universe to particles of small micro-world - the life of which only occupy a very small area. Life itself are at the top level, molecular is the lowest level. There is no characteristic of life down to the atomic level. Of course, it does not mean we will not introduce some of the parameters of atomic level when we describe the life status. In the development of theoretical physics, we know that the theory of relativity is about cosmoscopic level. It describes the system in high-speed movement of matter. Quantum mechanics is about micro level. It describes composition of matters and their relationship. Obviously, in the future architecture of modern medicine, there are macro and micro human body status description systems - based on modern medicine and TCM - similar to description levels of general relativity and quantum mechanics.

Under the guidance of complexity science and advancing on the road toward "precision medicine", the modern medical theory will integrate upward layer by layer whereas TCM will gradually analyze step by step downward. That is to say, to know the body structure, function, and the internal laws of the human body in order to achieve the objective of "precise" control, the two medical systems researchers are moving forward in an "orchestrated" effort from different directions. Since the focal points of the two medical systems in human body status description are far apart, they may not merge as one in the near future in the manner of physics studies which are united with "Unified Field Theory". But during the process of moving in from various directions, the two medical systems with their respective in-depth study of the body's rules and disease-control methodologies, undoubtedly that, their persistent orchestrated efforts will upgrade the future medical treatment and can significantly uplift the standard of health care.

In the field of life sciences, systems biology uses sophisticated methods and techniques of scientific research to study living systems. It is only a preliminary study using quantitative kinetic modeling approach to describe the dynamic behaviour of the system evolution. It is applicable to simple giant system. But it has great limitations and too many difficult problems to be solved when coming to complex adaptive systems. Therefore, scientists generally believe that the complexity of the organism systems is beyond our imagination. We should not study the whole organism system at this stage. Instead, we should study a certain part of the relatively independent and functional organism.

In the absence of advance science and technology, TCM establishes an effective system to treat and cure. In the wake of integrating biological systems, system biologists face the challenges and difficulties during the process. Can the biologists learn and be inspired by the successful examples of TCM and its methodology? Will they try to use TCM's holistic methodology to describe human body directly?

To establish a system that describes the human body status directly from holistic level, undoubtedly, can solve the problems of the huge amount of variables, great varieties and complexities. In the process of addressing the complexity of the system, are the adaptability and complex adaptive systems obstacles? Or, are they good assistants that will help us to take on the challenges? These are important methodological issues that modern medicine has to face on its path to Precision Medicine.

\section{Conclusion}

We portrayed the future position, relationship, and value of modern medicine and TCM in Precision Medicine in the context of complexity science. We drafted the basic structure of the integration of Western medicine and TCM in the future. There would be no difference between the two medical systems -Western medicine and TCM. In future medical system, it will be the difference between human body status description based on variables of holistic level, and human disease description based on variables of micro level. Precision Medicine developed based on TCM will focus on human body status description of holistic level. Precision Medicine developed based 
on Western medicine will focus on the revelation of human partial pathological changes and the precise treatment. With the development of medicine, TCM will advance into details downward where as modern medicine will integrate upward. Because of the complexities of the huge amount of human body components, wide variety, multilevel, and adaptability, the integration of Western medicine and TCM may be foreseeable or just within sight but will never meet.

In fact, although syndrome differentiation and treatment is the core of TCM, but clinical treatment also consists of disease differentiation syndrome (disorder), as well as giving consideration to both the incidental and fundamental specimens. Moreover, TCM practitioners make flawless prescriptions based on the integration of syndromestreatments and diseases (symptoms)-treatments. Thus, in the fusion of TCM and Western modern medicine system-treatment based on the holistic human body status description system and personalized medicine based on micro-level description system - can be combined in an organic manner. The integration of syndromes-treatments and diseases (symptoms)-treatments makes the best personalized medicine. With the fusion of TCM and Western medicine, modern medicine towards Precision Medicine, the treatment of human disease will become more accurate and more effective.

\section{Acknowledgments}

None.

\section{Conflicts of interest}

Author declares there are no conflicts of interest.

\section{Funding}

None.

\section{References}

1. Liyang WenIntroduction to Medicine. Beijing, China: People's Medical Publishing House; 2001. p.5-6.

2. Bing Yuan. An introduction to Modern Traditional Chinese Medicine. Beijing, China: People's Medical Publishing House; 2011. p.26.

3. Bing Yuan. Holistic Medicine - Integrated Chinese and Western Medicine Theory. Modern Medicine Press, Hong Kong, China. 2010.p.17.

4. Bing Yuan. Precision Medicine: A Complexity Science Perspective. Dalian, China: Medicine \& Philosophy; 2015 p. 4.

5. Http://news.cnstock.com/industry/sid rdjj/201511/3637940.htm

6. Bing Yuan. The Establishment of "Precise" Body States Control System - Traditional Chinese Medicine towards Precision (I), Beijing: Journal of Beijing University of Traditional Chinese Medicine. 2016;39(3):186190 .

7. Bing Yuan. The Establishment of "Precise" States Control System Traditional Chinese Medicine towards Precision (II). Journal of Beijing University of Traditional Chinese Medicine. 2016;39(4):4. 